\title{
A LONG SHORT TERM MEMORY APPLICATION ON THE TURKISH INTRADAY ELECTRICITY PRICE FORECASTING
}

\author{
DOI: 10.17261/Pressacademia.2018.867 \\ PAP- V.7-2018(20)-p.126-130
}

\section{Hakan Yorulmus ${ }^{1}$, Umut Ugurlu ${ }^{2}$, Oktay Tas $^{3}$}

${ }^{1}$ Istanbul Technical University, Management Engineering Deparment, Besiktas, Istanbul, Turkey. yorulmus@itu.edu.tr, ORCID: 0000-0002-8019-5308

${ }^{2}$ Istanbul Technical University, Management Engineering Deparment, Besiktas, Istanbul, Turkey. umut.ugurlu@itu.edu.tr, ORCID: 0000-0002-6183-969X

${ }^{3}$ Istanbul Technical University, Management Engineering Deparment, Besiktas, Istanbul, Turkey. oktay.tas@itu.edu.tr, ORCID: 0000-0002-7570-549X

To cite this document

Yorulmus, H., Ugurlu, U., Tas, O. (2018). A long short term memory application on the Turkish intraday electricity price forecasting. PressAcademia Procedia (PAP), V.7, p.126-130.

Permemant link to this document: $h$ ttp://doi.org/10.17261/Pressacademia.2018.867

Copyright: Published by PressAcademia and limited licenced re-use rights only.

\section{ABSTRACT}

Purpose- This paper aims to forecast the Turkish intraday electricity prices accurately. It will be the first intraday electricity price forecasting work, which uses Long-Short Term Memory (LSTM) application.

Methodology- LSTM method is based on a special kind of neural network, which is capable of learning long-term dependencies. This paper aims to achieve the best forecasts, in terms of Mean Absolute Error (MAE) and Root Mean Square Error (RMSE), by applying the LSTM model with multistep-ahead prediction approach.

Findings- LSTM model created in this study performed better with lagged values, electricity consumption and electricity production values. Especially using the lagged values of the prices and the reserve margin gave successful results.

Conclusion- The proposed method has improvement in the accuracy of forecasting. Turkish Intraday Electricity Market needs further research with time series methods as well as other neural network models

Keywords: Electricity price forecasting, Turkish intraday electricity market, LSTM, multistep-ahead prediction, deep learning, time series. JEL Codes: C45, Q47, G17

\section{INTRODUCTION}

Electricity price forecasting plays a vital role for many of the electricity-related companies. Both energy providers and consumers need to forecast electricity prices accurately to prevent loss of profits. Electricity prices are forecasted in many different horizons from intraday markets to the long-term bilateral contracts. They are also forecasted by using various methods. According to Weron (2014), electricity price forecasting methods can be gathered under five main topics: Multi-agent models, fundamental models, reduced-form methods, statistical methods and the computational intelligence methods. In addition to them hybrid methods are also popular and relatively successful compared to the individiual methods. Moreover, Lasso regression and other types of dimension reduction methods as well as automated variable selection (Uniejewski et al., 2016) applications are quite popular. However, all these methods are applied in the dayahead market and applications on the intraday prices are very limited.

Although day-ahead market and the intraday market have some similarities, difficulties have a paramount effect in the context of forecasting. First of all, forecasts are generally from $12 \mathrm{~h}$ to $36 \mathrm{~h}$ in the day ahead market. In the intaday market generally forecasts are made for 1 hour ahead. If there is more than one hour between the auction closing hour and the delivery like in the Turkish market (1.5 hour), then forecasts must be done for 2 hour ahead. This phenomena changes all the variables used in the electricity price forecasting process. Realized lagged prices from 1 hour on can be used in the intraday electricity price forecasting.

Turkish electricity market is an improving market. Although $75-80 \%$ of the electricity trade is still done by the bilateral contracts. About 15 $20 \%$ of the energy is sold in the Turkish day-ahead market. Even though share of the trades in the balancing market, which is between dayahead and intraday markets chronologically, is still very low, the share of the intraday market increased up to $5 \%$ of all the trades. With the improvement of the intraday market, energy derivatives are also started to be priced and traded, for both hedging and speculative purposes. The installed capacity of the hydro generation is relatively high by $34.3 \%$ and the share of the wind energy production share increase day by day, which is $7.6 \%$ in 2016 (EPIAS, 2016). Although all the electricity markets are unique, Turkish electricity market has similarities with the other Mediterranean markets such as Spanish one (Diaz and Planas, 2016) and the other Southeastern markets (Hryrshchuk and Lessmann, 2018). 
Nowadays deep neural networks have become popular in many fields. Electricity price forecasting has become one of these fields with the applications of Lago et al. (2018), Ugurlu et al. (2018), Kuo and Huang (2018). Especially recurrent neural networks, which are mainly Long short term memory (LSTM) and Gated recurrent units (GRU), are tailor-made for the time-dependent problems due to their ability of capturing the long term time dependencies. These deep learning algorithms are especially successful, when high numbers of data is provided.

The purpose of our paper is to forecast the intraday electricity prices accurately. For this purpose, we will use the Turkish intraday electricity prices and train our data in an LSTM neural networks, then we will forecast intraday electricity prices in a 2-step-ahead forecast scheme. The errors of our forecasts will be calculated with various forecast performance measures and the forecast performance of our model will be evaluated. We will also examine the effect of adding more variables and compare the forecast performance of changing exogenous variables. The contribution of this paper is two fold: Firstly, it is the first LSTM application in the intraday electricity price forecasting. Secondly, it is the first deep learning application in the intraday electricity markets.

\section{LITERATURE REVIEW}

Day-ahead electricity price forecasting has a widened literature with many papers from many applications by researchers from various areas. However, there are only a few papers on the intraday electricity prices and even less on the forecasting issue. Kiesel and Paraschiv (2017) investigates the bidding behaviour in the German intraday market by focusing on the wind and photovoltaic forecasts as well as reserve margin. According to authors, intraday prices reflect asymmetrically to the forecast errors in renewables. Mærkle-Huß et al. (2018) also mention the delivery duration of 15 minutes, which is decreased from 1 hour. They mention that this is to balance the intra-hour volatility of the renewables. It mentions that decreasing the delivery time could have a positive effect on the market-makers. Work of Panagiotelis and Smith (2008) forecast the intraday electricity prices in terms of Bayesian density forecasting and by using the multivariate skew $t$ distributions. They have a 30 day forecast period and esimate the model with the Markov Chain Monte Carlo. Then, they forecast the electricity prices by different distributions. Klæboe et al. (2015) perform 1 hour ahead forecasts fro many models in the intraday market. Their main finding is that the day-ahead electricity prices have a very crucial effect on the balancing market prices. Holm (2017) investigates the price determinants in the Nordpool intraday market. High trading activity, imbalance costs and the amount of generated power have significant effects on the trading behaviour. As we also examine in the Turkish intraday market, most of the trades occur nearer to the gate closure. In the regression spot price and regulating power price are used as the independent variables and their model have an overall good forecast ability in relatively calmer periods. However, it struggles in the price spikes. The model is clearly effected by the seasonality in different time frequencies as well. Hagemann (2013) has very similar finding. He investigates the effects of power plant outages, wind and power productions' forecast errors, load forecast errors, foreign demand and supply are investigated. Similar finding to Holm (2017) is that the price determinants vary according to seasonality. Sharff and Amelin (2016) and Pape et al. (2016) investigate the price determinants in the Nordpool and German EPEX market, respectively. Moreover, Monteiro et al. (2016) forecast the Iberian Intraday market prices. There are various groups of forecast zones, which we call auction based intraday-market (Hagemann and Weber, 2015), and they forecast the intraday prices for these sessions.

Deep learning algorithms are only used in three very recent papers in the electricity price forecasting literature. All these papers work on the day-ahead prices. Ugurlu et al. (2018) apply deep LSTM and deep GRU networks and compare them with the shallow networks as well as statistical methods and neural networks models are significantly better than the statistical methods. Furthermore, adding more variables have a positive effect on the forecast performance, especially when the number of the layers in the networks is increased. On the other hand, Lago et al. (2018) apply more models, which can be used as a benchmark in the electricity price forecasting literature. Furthermore, they also propose hybrid deep learning models. Kuo and Huang (2018) propose both Convolutional neural networks (CNN) and LSTM as deep network structures. According to their results, combining both methods as a hybrid method, give better results than the individual ones.

\section{DATA AND METHODOLOGY}

Motivation of this research is finding better variables and model for making better preditiction of electricity price in intraday electricity market. The data set for this research was obtained from Energy Exchange Istanbul Transpercy Platform. While forming data 1000 observation during the period of 2017/02/08 07:00 - 2018/03/31 22:00 was used. All observation is hourly data with 16 variables. Avarage price is dependent variable of model. In transpercy platform, observers can see hourly contracts according to electricity price. In an hour lots of contracts can be offered. Average Price variable is obtained from fulfilled contracts prices according to their lots. According their lot size with weighted average method, average price variable was created hourly.

\section{Table 1: Data Definitions}

\begin{tabular}{lll}
\hline Variable & Description & Range \\
\hline V1 & Average Price & $5,7-625(\mathrm{TL} / \mathrm{MWh})$ \\
V2 & 1 Hour Lagged Average Price & $5,7-625(\mathrm{TL} / \mathrm{MWh})$ \\
V3 & 2 Hours Lagged Average Price & $5,7-625(\mathrm{TL} / \mathrm{MWh})$ \\
V4 & 3 Hours Lagged Average Price & $5,7-625(\mathrm{TL} / \mathrm{MWh})$ \\
V5 & 24 Hours Lagged Average Price & $5,7-625(\mathrm{TL} / \mathrm{MWh})$ \\
V6 & 48 Hours Lagged Average Price & $5,7-625(\mathrm{TL} / \mathrm{MWh})$ \\
V7 & 72 Hours Lagged Average Price & $5,7-625(\mathrm{TL} / \mathrm{MWh})$ \\
V8 & 168 Hours Lagged Average Price & $5,7-625(\mathrm{TL} / \mathrm{MWh})$ \\
V9 & Month & $1(\mathrm{Jan})-12(\mathrm{Dec})$ \\
V10 & Hour & $0-23$
\end{tabular}




\begin{tabular}{lll} 
V11 & Total Final Daily Production Program & $17650-42814(\mathrm{MWh})$ \\
V12 & Total Real-Time Generation & $18850-446990(\mathrm{MWh})$ \\
V13 & Load Forecast & $0-46500(\mathrm{MWh})$ \\
V14 & Consumption & $18850-47062(\mathrm{MWh})$ \\
V15 & Positive Imbalance Cost & $155-3490(\mathrm{TL})$ \\
V16 & Negative Imbalance Cost & $(-4858)-(-144)(\mathrm{TL})$ \\
\hline
\end{tabular}

As seen in Table 1, average electiricity price and 1,2,3,24,48,72,168 lagged average prices of electricity was selected for seen effect on LSTM model. Also month and hour variables are for decreasing effect of seasonality and certain days or months effect. Moreover, production, consumption and imbalance cost variables are added to increase model performance. Maximum and minimum values of variables are seen in Table 1.

Using time series prediction methods allows us to only using values, which are obsereved in the past. In multistep ahead time series sequence of future values gives us to find properties variability, frequency of abnormally high or low values etc. Multistep-ahead time series prediction used some examples are shown in literature to find stock prices, traffic volume, and electrical power consumption. Common method a single model from past values of the time series and begins the model step by step to predict its future values. This is multi-stage prediction which causes some problems such as accumulation of error problem. It leads to acculate erros the past error predictions to future predictions. In this paper multistep-ahead time series prediction with independent value prediction is selected for implemantation. This model builds a separate model for each prediction step using only its past observations. In independent value prediction method for each step seperate models are used for each model has the same input $\mathrm{X}$ but different output $\mathrm{Y}$. This aspect of method is helpful for avoiding error accumulation problem which is encountered with multi-stage step prediction (Cheng, 2006).

We implement LSTM model with indirect multistep-ahead prediction method to find better prediction of Turkish intraday electricity price. LSTM is a type of recurrent neural network used in deep learning. LSTMs have been used to advance the state-of the-art for many difficult problems. LSTMs are the special type of Recurrent Neural Network (RNN) which are capable of learning long term dependencies. LSTMs avoid long-term dependency problem. Model not struggled to learn, basicly remembers information for long period of time default behaviors (Greff, 2017). Main idea of LSTM memory cell is maintain its state over time, and non-linear gating units that regulate the information flow into and out of the cell. Basiclly LSTM cells have input output and forget gate. And also multiple cell combinations are possible. Forget gate allows the model to learn continuously. LSTM like all recurrent neural networks has chain of repeating modules of neural networks. In our paper, we use two layers both LSTMs for multistep-ahead time series model predict 2 hours ahead with two steps.

\section{FINDINGS}

In this paper, we use time series prediction with LSTM neural network multi steps ahead aproach with independent value prediction approach for Turkish intraday electricity price data. LSTM is one of the deep learning methods we combine it with with this approach a separate model is built for each prediction step. Because of more than one hour between the auction-closing hour and the delivery like in the Turkish market (1.5 hour), forecasts must be done for 2 hour ahead. For this time series prediction the number of steps to predict 2 hours ahead and 2 LSTM models built with Keras in python. This approach provide us to avoid any memory leakage between models.

Table 2: LSTM Models for 2 Hours Ahead Prediction and Their Evaluation Metrics

\begin{tabular}{|c|c|c|c|c|c|c|c|c|c|c|c|c|}
\hline & IVI & IVIL & IVIS & 1014 & IVIO & 1010 & IVII & 1010 & IVIY & IVIO & IVII & IVI12 \\
\hline Avarege Price & $x$ & $\mathrm{X}$ & $x$ & $\mathrm{X}$ & $X$ & $\mathrm{x}$ & $\mathrm{X}$ & $\mathrm{X}$ & $x$ & $\mathrm{X}$ & $\mathrm{x}$ & $\mathrm{X}$ \\
\hline 1 Hour Lagged Avarage Price & $x$ & & $x$ & $x$ & $x$ & $x$ & $x$ & $x$ & $x$ & $x$ & $\mathrm{x}$ & \\
\hline 2 Hours Lagged Avarage Price & $x$ & & $x$ & $x$ & & & & & & $x$ & $x$ & \\
\hline 3 Hours Lagged Avarage Price & $x$ & & $x$ & $x$ & & & & & & $x$ & $x$ & \\
\hline 24 Hours Lagged Avarage Price & $x$ & $\mathrm{X}$ & $x$ & & $X$ & $\mathrm{x}$ & $\mathrm{X}$ & $\mathrm{X}$ & $x$ & $\mathrm{X}$ & $\mathrm{X}$ & $\mathrm{X}$ \\
\hline 48 Hours Lagged Avarage Price & $x$ & $x$ & $x$ & & $x$ & $x$ & $x$ & $x$ & $x$ & & & \\
\hline 72 Hours Lagged Avarage Price & $\mathrm{X}$ & $\mathrm{X}$ & $x$ & & $\mathrm{X}$ & $\mathrm{x}$ & $\mathrm{X}$ & $x$ & $x$ & & & \\
\hline 168 Hours Lagged Avarage Price & $x$ & $x$ & $x$ & & $x$ & $x$ & $\mathrm{X}$ & $\mathrm{x}$ & $x$ & & & \\
\hline Month & $\mathrm{x}$ & & & & $x$ & $\mathrm{x}$ & $\mathrm{x}$ & $x$ & $x$ & $\mathrm{X}$ & $\mathrm{X}$ & $x$ \\
\hline Hour & $x$ & & $\mathrm{X}$ & $\mathrm{X}$ & $\mathrm{x}$ & $x$ & $\mathrm{X}$ & $\mathrm{X}$ & $x$ & $x$ & $\mathrm{x}$ & $x$ \\
\hline Total Final Daily Production Program & $x$ & & & $x$ & $\mathrm{x}$ & $\mathrm{x}$ & $\mathrm{x}$ & $x$ & $x$ & $x$ & & $x$ \\
\hline Total Real-Time Generation & $\mathrm{x}$ & & & $x$ & & $\mathrm{x}$ & $\mathrm{X}$ & $\mathrm{x}$ & $x$ & $x$ & & $x$ \\
\hline Load Forecast & $\mathrm{x}$ & & & $x$ & & & $\mathrm{x}$ & $x$ & $x$ & $x$ & & $x$ \\
\hline Consumption & $x$ & & & $x$ & & & & $x$ & & $x$ & & $x$ \\
\hline Positive Imbalance Cost & $\mathrm{x}$ & & & $\mathrm{x}$ & & & & & $X$ & $x$ & & $x$ \\
\hline Negative Imbalance Cost & $\mathrm{x}$ & & & $\mathrm{x}$ & & & & & $\mathrm{X}$ & $\mathrm{X}$ & & $\mathrm{X}$ \\
\hline MAE & 17.2 & 18.4 & 20.0 & 44.0 & 50.3 & 19.5 & 19.2 & 27.3 & 18.2 & 24.4 & 18.6 & 28.1 \\
\hline MAPE & 0.24 & 0.22 & 0.26 & 0.56 & 0.49 & 0.23 & 0.25 & 0.32 & 0.22 & 0.32 & 0.25 & 0.32 \\
\hline RMSE & 25.06 & 26.0 & 27.7 & 57.2 & 57.54 & 27.01 & 26.78 & 35.75 & 25.5 & 32.6 & 26.4 & 36.0 \\
\hline $\mathbf{R}^{\wedge} \mathbf{2}$ & 0.45 & 0.40 & 0.32 & -1.88 & -1.91 & 0.35 & 0.36 & -0.12 & 0.42 & 0.06 & 0.38 & -0.14 \\
\hline
\end{tabular}

Two LSTM models are built one of them is for 1-hour ahead prediction other is for 2 hours ahead prediction. Both LSTM model have two layers for avoiding any overfitting problem. During the 2017/02/08 07:00 - 2018/03/31 22:00 period there is 10000 observations, 7000 are 
selected for training set 1000 observation for validation set and 2000 observation for testing. With validation set proper parameters are selected. MAE, MAPE values are compared with traninig set and validation set, understood best parameters are selected and they avoided from overfitting problem. For first LSTM model first layer units size 320, second layer units size 60, batch size 150, epoch value 150 selected and for activation tanh and inner activation hard sigmoid functions selected with adam optimizer. In addition, second LSTM model first layer units size 200 , second layer units size 60 , batch size 80 , epoch value 15 selected and for activation tanh and inner activation hard sigmoid functions selected with adam optimizer.

After creating model and adjusted, variable combinations are tested. In our case for intra day electricity price forecast two hours ahead prediction is more importand than one hour ahead. Because of this reason, two ahead electricity price forecast LSTM model values are given in Table 2. According to table 2 best model for two hours ahead prediction is first model with variables; average price, $1,2,3,24,48,72,168$ lagged average price, month, hour, total final daily production program, total real-time generation, load forecast, consumption, positive imbalance cost, negative imbalance cost. This model has 17,2 TL MAE value which is good value fort his kind of market prediction and $r$ square value is better than other models. Moreover, when compared models with each other, for 2 ahead prediction lag values gives better performance than production and consumption variables but when all variables together became better performanced LSTM model.

\section{CONCLUSION}

The Turkish intraday electricity prices forcasting is realy challenging task and also forecasting two hours ahead price has some problems. In our paper, train our data in an LSTM neural networks with multistep-ahead prediction approach, then we will forecast intraday electricity prices in a 2-step-ahead forecast scheme. The errors of our forecasts will be calculated with various forecast performance measures and the forecast performance of our model will be evaluated. We will also examine the effect of adding more variables and compare the forecast performance of changing exogenous variables. Using LSTM model with two layers and adding all variables which is selected variables performed moderate performance with 17,2 TL MAE value. On the other hand, 17 TL error is good metric according to this type of market.This paper, it is the first LSTM application in the intraday electricity price forecasting. Secondly, it is the first deep learning application in the intraday electricity markets. In the further research, direct multistep-ahead prediction method, new deep learning methods and new variables can be used for precise forecasting.

\section{REFERENCES}

Cheng, H., Tan, P. N., Gao, J., Scripps, J. (2006, April). Multistep-ahead time series prediction. In Pacific-Asia Conference on Knowledge Discovery and Data Mining (pp. 765-774). Springer, Berlin, Heidelberg.

Díaz, G., Planas, E. (2016). A note on the normalization of Spanish electricity spot prices. IEEE Transactions on Power Systems, 31(3), 24992500.

EPIAS (2016). Annual Report. 01 January 2016 - 31 December 2016.

Greff, K., Srivastava, R. K., Koutník, J., Steunebrink, B. R., Schmidhuber, J. (2017). LSTM: A search space odyssey. IEEE transactions on neural networks and learning systems, 28(10), 2222-2232.

Hagemann, S. (2013). Price determinants in the German intraday market for electricity: an empirical analysis.

Hagemann, S., Weber, C. (2015). Trading volumes in intraday markets: theoretical reference model and empirical observations in selected European markets (No. 03/15). EWL working paper.

Holm, T. B. (2017). The future importance of short term markets: an analyse of intraday prices in the Nordic intraday market; Elbas (Master's thesis, Norwegian University of Life Sciences, Ås).

Hryshchuk, A., Lessmann, S. (2018). Deregulated day-ahead electricity markets in Southeast Europe: price forecasting and comparative structural analysis.

Kiesel, R., Paraschiv, F. (2017). Econometric analysis of 15-minute intraday electricity prices. Energy Economics, 64, 77-90.

Klæboe, G., Eriksrud, A. L., Fleten, S. E. (2015). Benchmarking time series based forecasting models for electricity balancing market prices. Energy Systems, 6(1), 43-61.

Kuo, P. H., Huang, C. J. (2018). A high precision artificial neural networks model for short-term energy load forecasting. Energies, $11(1)$, 213.

Lago, J., De Ridder, F., De Schutter, B. (2018). Forecasting spot electricity prices: deep learning approaches and empirical comparison of traditional algorithms. Applied Energy, 221, 386-405.

Märkle-Huß, J., Feuerriegel, S., Neumann, D. (2018). Contract durations in the electricity market: causal impact of $15 \mathrm{~min}$ trading on the EPEX SPOT market. Energy Economics, 69, 367-378.

Monteiro, C., Ramirez-Rosado, I. J., Fernandez-Jimenez, L. A., Conde, P. (2016). Short-term price forecasting models based on artificial neural networks for intraday sessions in the iberian electricity market. Energies, 9(9), 721.

Panagiotelis, A., Smith, M. (2008). Bayesian density forecasting of intraday electricity prices using multivariate skew $t$ distributions. International Journal of Forecasting, 24(4), 710-727. 
Pape, C., Hagemann, S., Weber, C. (2016). Are fundamentals enough? Explaining price variations in the German day-ahead and intraday power market. Energy Economics, 54, 376-387.

Scharff, R., Amelin, M. (2016). Trading behaviour on the continuous intraday market Elbas. Energy Policy, 88, $544-557$.

Ugurlu, U., Oksuz, I., Tas, O. (2018). Electricity price forecasting using recurrent neural networks. Energies, $11(5), 1255$.

Uniejewski, B., Nowotarski, J., Weron, R. (2016). Automated variable selection and shrinkage for day-ahead electricity price forecasting. Energies, 9(8), 621.

Weron, R. (2014). Electricity price forecasting: a review of the state-of-the-art with a look into the future. International journal of forecasting, 30(4), 1030-1081. 